\title{
Arthroscopic treatment for posteromedial talar process fracture (Cedell fracture): a case report
}

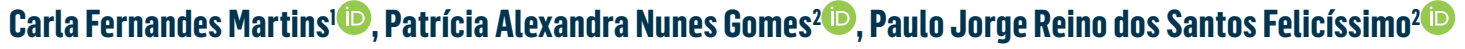 \\ 1. Orthopedic Surgeon, Department of Orthopedics, Centro Hospitalar Tondela - Viseu, Viseu, Portugal. \\ 2. Orthopedic Surgeon, Department of Orthopedics, Hospital CUF Descobertas, Lisboa, Portugal.
}

\begin{abstract}
Fracture of the posteromedial talar process (Cedell fracture) is a rare injury and is easily misdiagnosed as a simple ankle sprain. Suspicion should be heightened if specific mechanisms of injury are present, and, in these cases, a CT scan should be performed. Significant ankle pain and disability can result if these injuries are not identified and treated properly. Few cases have been described in the literature, and the efficacy of surgical techniques and approaches for fractures of the posterior talar process remains controversial. We report a 56-year-old man with an acute posteromedial talar fracture treated arthroscopically. This approach provides good access to the posterior ankle compartment, subtalar joint, and extraarticular structures, which may allow fracture reduction and fixation under arthroscopic visualization.
\end{abstract}

Level of Evidence V; Therapeutic Studies; Expert Opinion.

Keywords: Talus; Fractures, bone; Arthroscopy; Fracture fixation, internal.

\section{Introduction}

Talar fractures constitute only 3\%-5\% of all reported foot and ankle fractures, and by far the rarest fractures are those of the posterior talar process ${ }^{(1)}$. The posterior process of the talus consists of lateral and medial tubercles, with the groove for the flexor hallucis longus (FHL) tendon located in the middle between the two tubercles. The inferior surface forms the posterior $25 \%$ of the subtalar joint, being essential for normal subtalar movement ${ }^{(2)}$. Fractures of the posterior process of the talus, and, in particular, those of the posteromedial process are extremely rare, with few cases reported in the literature ${ }^{(1,3,4)}$. The first descriptions on medial tubercle fractures are attributed to Cedell(5) in 1974. Cedell described the posteromedial tubercle fracture as an avulsion injury when the foot is pronated and is forcibly flexed dorsally, increasing tension in the posterior tibiotalar ligament ${ }^{(3,6)}$. Certain reports also indicated that this injury might occur when the supinated foot is forcefully plantarflexed ${ }^{(1,7)}$. Alternative proposed mechanisms include impingement of the sustentaculum tali and direct trauma to the posteromedial facet ${ }^{(3,6)}$. On physical examination, patients present with pain and swelling in the hindfoot, usually with pain increasing with passive extension of the big toe (positive posterior talar impingement test) ${ }^{(6)}$. Up to $40 \%$ of these fractures may be missed at initial presentation because they are rare and resemble an ankle sprain on standard radiographs ${ }^{(6)}$. Suspicion should be maintained based on specific mechanisms of injury. A CT scan should be performed to identify the fracture and assess displacement and fragment size ${ }^{(4,6)}$.

Incorrect and delayed diagnosis may be responsible for painful nonunion, posterior ankle impingement, tarsal tunnel syndrome, subtalar arthritis, or FHL entrapment ${ }^{(2)}$. The choice of optimal treatment method for posteromedial talar process fractures is unclear, and depends on subtalar joint involvement, fragment size, degree of displacement, and comminution $^{(2,6)}$. Various treatments for this injury are advocated in the literature: conservative treatment of minimally or nondisplaced fractures, with non-weight-bearing cast immobilization for 4 to 6 weeks; fracture excision in painful nonunion, highly comminuted fractures, or malunions causing posteromedial ankle impingement ${ }^{(7,8)}$; primary open reduction and internal fixation (ORIF), which is recommended for the majority of $\mathrm{Ce}$ dell fractures that are less than 4 weeks old, with fracture fragments $>1 \mathrm{~cm}$, displaced $(>2 \mathrm{~mm}$ ) and with disruption of the

Study performed at the Orthopedic Surgeon, Department of Orthopedics, Hospital CUF Descobertas, Lisboa, Portugal.

Correspondence: Carla Fernandes Martins. Av. Rei Dom Duarte - Centro Hospitalar Tondela-Viseu, 3504-509, Viseu, Portugal. E-mail: martins.f.carla@gmail.com. Conflicts of interest: none. Source of funding: none. Date received: October 25, 2021. Date accepted: December 09, 2021. Online: December 20, 2021.

How to cite this article: Martins CF, Gomes

PAN, Felicíssimo PJRS. Arthroscopic treatment for posteromedial talar process fracture (Cedell fracture): a case report. J Foot Ankle. 2021;15(3):273-7.

\section{(i) (8)}


subtalar joint ${ }^{(6)}$; arthroscopic approaches for screw fixation or excision of the fragment have also been described(3,8-10). The purpose of this article is to raise physician suspicion of this uncommon fracture and present a case of arthroscopy-assisted reduction and screw fixation of acute posteromedial talar fracture.

\section{Case description}

Ethics Committee approval and written consent from the patient were obtained for this report.

A 56-year-old male presented to our hospital complaining of right ankle pain and swelling after a fall from stairs. To his recollection, the foot was in plantarflexion and supination at the time of injury. The patient's medical and surgical history was unremarkable, and there were no previous injuries to the right lower extremity. Physical examination revealed significant swelling and severe tenderness of the ankle joint. There was no neurovascular compromise. Ecchymosis was present over the posteromedial aspect of the ankle, spreading to the foot, along the path of the FHL (Figure 1), and pain occurred behind the medial malleolus during passive motion of the hallux. Standard radiographs of the ankle raised suspicion of the presence of a small fragment on the posterior aspect of the talus (Figure 2). A CT scan of the right ankle was perfor-

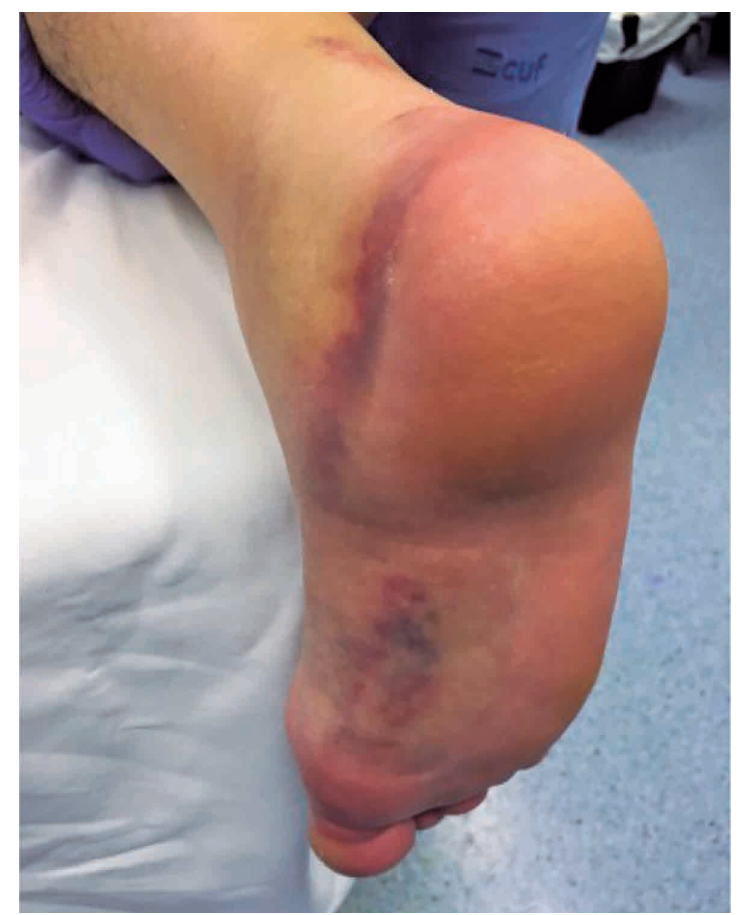

Figure 1. Patient's right foot and ankle. Swelling of the ankle joint and bruising over the posteromedial aspect of the ankle spreading to the foot, in the path of the flexor hallucis longus tendon sheath. This bruise trajectory could be a potential diagnostic sign of Cedell fracture. med for more detailed examination and evaluation. A fracture of the posteromedial tubercle of the talus was found; the size of the fragment was approximately $2.2 \times 0.9 \times 1.8 \mathrm{~cm}$, with a 3-mm displacement (Figure 3). A posterior hindfoot arthroscopy was performed. The patient was placed in the prone position and a tourniquet was applied to the right thigh. The procedure was performed through the posterolateral and posteromedial portals. The FHL tendon was visualized and retracted laterally to expose the posteromedial talar process and fracture (Figure 4A). The surrounding inflamed synovium was resected. The fracture was reduced and fixed with a guidewire (Figure 4B). The position of the guidewire was checked with fluoroscopy (Figure 4C-D). A cannulated screw of appropriate length was inserted (Figure 4E-F). The ankle was plantarflexed and the hallux dorsiflexed to ensure that ankle motion and sliding of the $\mathrm{FHL}$ were not impeded by the screw head. The position of the screw was verified by fluoroscopy (Figure 5). In the postoperative period, the foot was immobilized in a short leg cast for 4 weeks (Figure 6). At this time, the patient began physical therapy but maintained immobilization with a non-weight-bearing walking boot for an additional 6 weeks. The swelling gradually subsided, and the patient reported no pain in the ankle or during hallux dorsiflexion.

\section{Discussion}

There have been few reports of Cedell fracture in the literature ${ }^{(3)}$. This fracture is most commonly described as an avulsion injury resulting from a pronation-dorsiflexion force causing tension at the insertion of the posterior talotibial ligament. Most cases have been attributed to this indirect effect $t^{(1,3,5)}$. Nevertheless, the injury may occur as a result of an inversion and a forced plantarflexion mechanism leading to direct compression of the posterior talus, as seems to have occurred in our patient ${ }^{(6,7)}$. This mechanism of injury on landing may explain the vertical compressive force and splitting
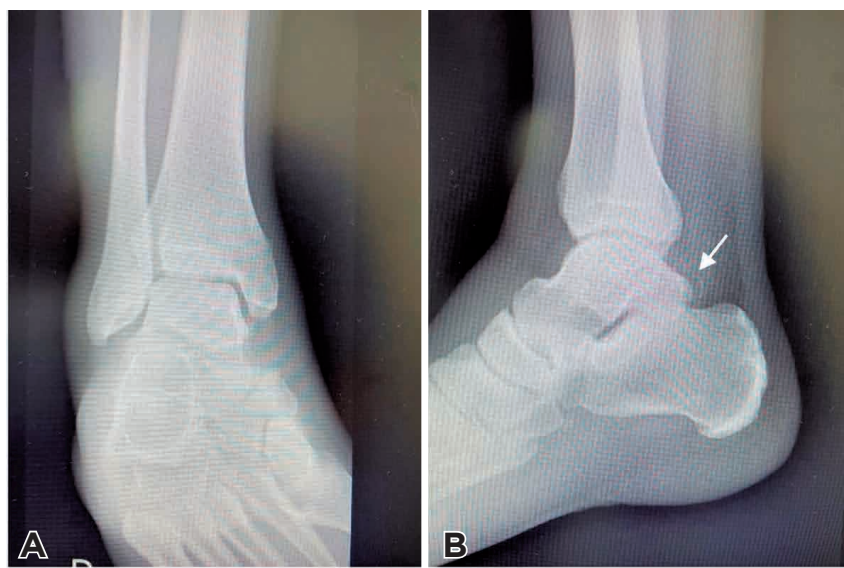

Figure 2. Preoperative A) anteroposterior and B) lateral radiographs of the right ankle, showing suspicious findings on the posteriomedial aspect of the talus (arrow). 

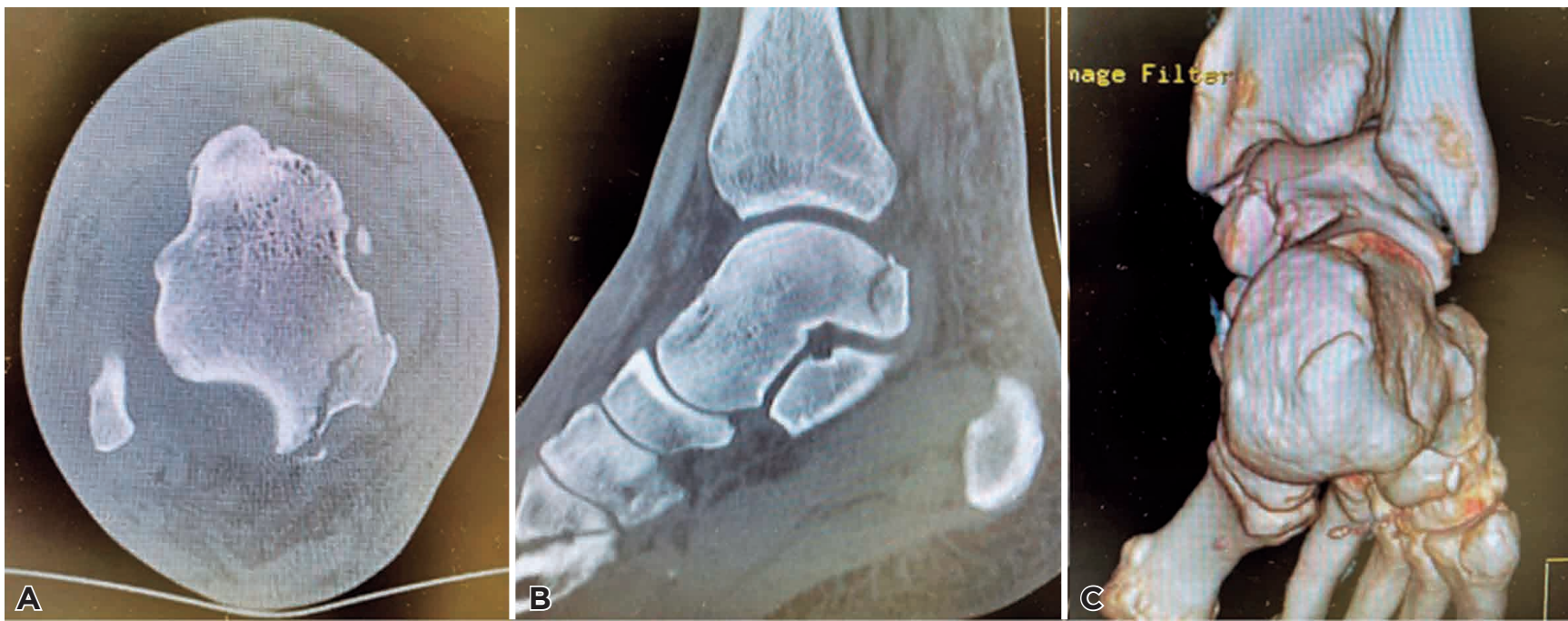

Figure 3. Preoperative computed tomography showing a displaced posteromedial talus fracture (arrow). A) Transverse view; B) sagittal view; C) 3-dimensional reconstruction.
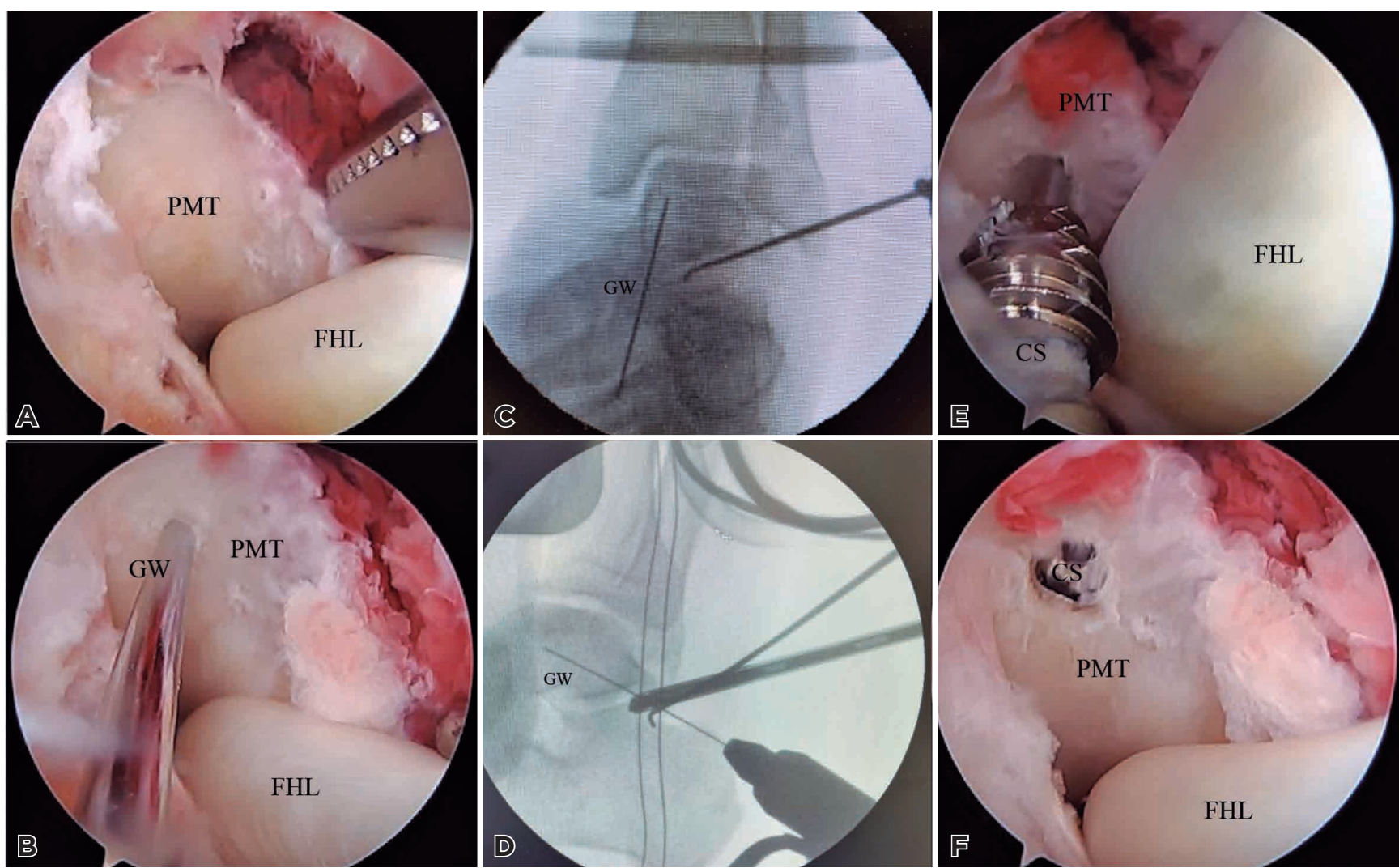

Figure 4. Endoscopically assisted reduction and screw fixation of acute fracture of the posteromedial talar process of right ankle. A) Arthroscopic view of the posteromedial talus fracture from the posterolateral portal. The FHL tendon is retracted laterally for better fracture exposure; B) Fracture reduced and temporarily fixed with a guidewire. The position of the guidewire is checked with fluoroscopy [anteroposterior view C), lateral view D)]; E,F) Placement of cannulated screw. PMT, posteromedial talar process; FHL, flexor hallucis Longus tendon; GW, guidewire; CS, cannulated screw. 


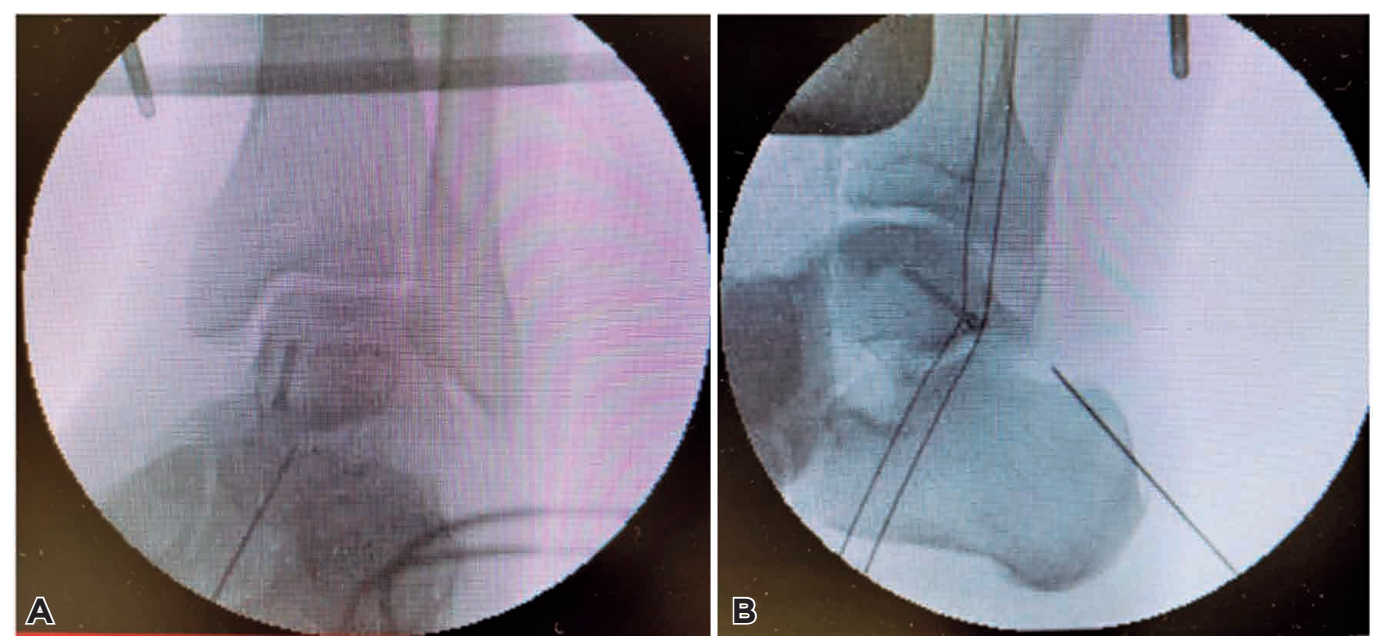

Figure 5. Endoscopically assisted reduction and screw fixation of acute fracture of the posteromedial talar process of right ankle. A) Position of the screw checked with fluoroscopy. Anteroposterior view; B) lateral view.
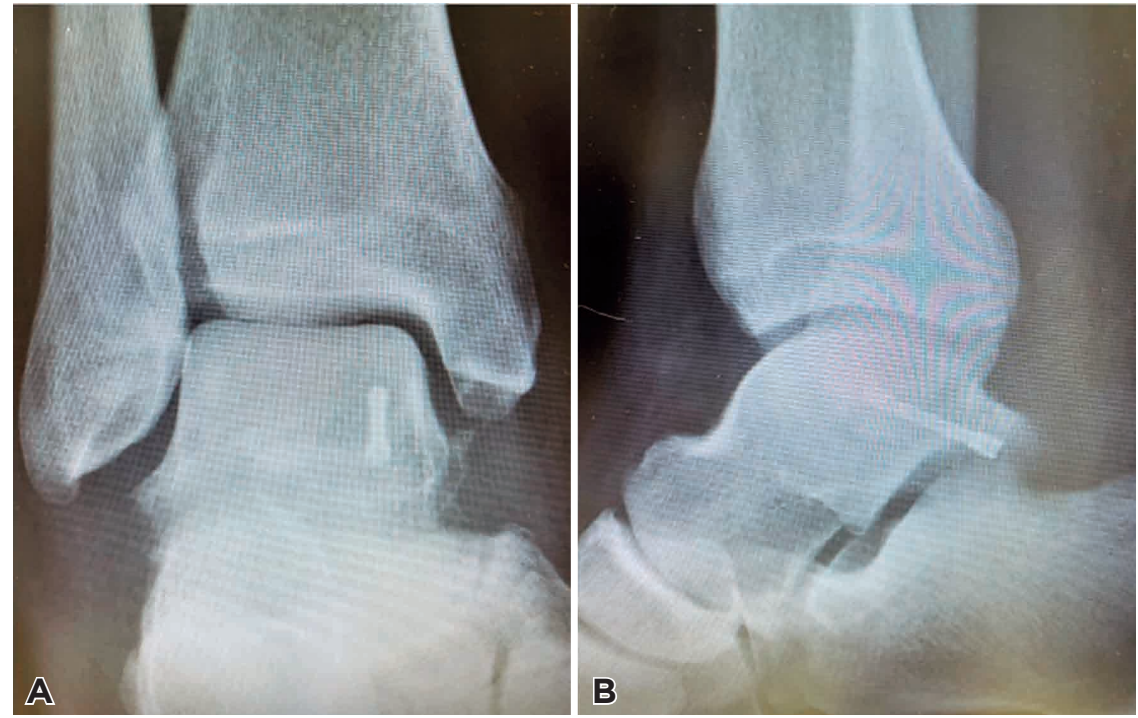

Figure 6. Postoperative radiographs obtained at 4-week follow-up. A) Anteroposterior and $\mathrm{B}$ ) lateral radiographs of the right ankle.

of the posterior talar process at the base of the posteromedial tubercle in the present case. These fractures could be misdiagnosed as severe ankle sprains, mostly due to poor visualization on routine radiographs. Timely diagnosis and treatment of such an injury is of great importance in restoring subtalar joint anatomy and function, as minimal displacement can lead to posttraumatic arthritis, painful nonunion, or posterior impingement ${ }^{(2,8,9)}$. The real challenge is to choose appropriate treatment after recognizing a Cedell fracture. To the author's knowledge, and according to a recent review, there have been few descriptions of surgical techniques for this type of fracture, consisting mainly of case reports ${ }^{(3)}$. Most of these publications also lack a detailed description of the patients and their management ${ }^{(3,4)}$. In general, there are three strategies for treatment of Cedell fractures: conservative treatment with immobilization alone; excision of the fragment; or osteosynthesis, which may be performed by open or arthroscopic approaches ${ }^{(3,4,8-10)}$. The optimal treatment for fractures of the posteromedial talar process is still unclear, and depends on the fracture pattern. Large, displaced fragments with joint involvement and subtalar dislocation often result in significant incongruity of the subtalar joint and require surgical intervention ${ }^{(2)}$. Some complications, especially wound healing problems, are associated with open surgical approaches 
to the hindfoot due to poor blood supply in this region ${ }^{(10)}$. Minimally invasive methods have been developed to reduce surgical trauma. Nevertheless, percutaneous screw fixation can be difficult as a minimally invasive surgical approach. The posterior screw may injure the posterior subtalar joint, the FHL tendon, and the tibial neurovascular bundle ${ }^{(8)}$. The posterior arthroscopic approach is proving to be an interesting treatment alternative. To our knowledge, only a few cases are known in which a posteromedial talar fracture has been treated arthroscopically, and the majority of these are arthroscopic excision of avulsion fragments ${ }^{(3,8-10)}$. We demonstrate, in our case, that such fractures can be reduced under arthroscopic visualization and that screw insertion is facilitated by mobilization of the FHL tendon. The procedure provided excellent access to the posterior ankle compartment and a better visualization of the fracture fragments and the subtalar joint, which allowed reduction and screw fixation as well as correction of the posterior ankle impingement. The advantage of this minimally invasive technique is less soft- tissue trauma, fewer wound complications, shorter recovery time, and a better cosmetic outcome ${ }^{(8,9)}$. In our opinion, internal fixation with hindfoot arthroscopy can be a safe treatment option for posteromedial talar fracture, when performed by an experienced arthroscopist.

\section{Conclusion}

This case demonstrates that arthroscopy-assisted reduction and screw fixation is a viable alternative technique for treatment of posteromedial talar fractures.

Authors' contributions: Each author contributed individually and significantly to the development of this article: CFM *(https://orcid.org/OOOO-OOO18454-3050) Conceived and planned the activities that led to the paper, wrote the paper, participated in the bibliographic review, in the reviewing process, approved the final version; PANG *(https://orcid.org/0000-0002-1681-109X) Conceived and planned the activities that led to the paper, participated in the reviewing process, approved the final version; PJRSF *(https://orcid.org/000-0003-1892-8360) Conceived and planned the activities that led to the paper, performed the surgery, participated in the reviewing process, approved the final version. All authors read and approved the final manuscript. *ORCID (Open Researcher and Contributor ID) iD).

\section{References}

1. Letonoff EJ, Najarian CB, Suleiman J. The posteromedial process fracture of the talus: a case report. J Foot Ankle Surg. 2002; 41(1):52-6.

2. Hsu AR, Scolaro JA. Posteromedial Approach for Open Reduction and Internal Fixation of Talar Process Fractures. Foot Ankle Int. 2016;37(4):446-52.

3. Zwiers R, de Leeuw PAJ, Wiegerinck EMA, van Dijk CN. Surgical treatment for posteromedial talar process fractures. Foot Ankle Surg. 2020;26(8):911-7.

4. Engelmann EWM, Wijers O, Posthuma JJ, Schepers T. Systematic review: Diagnostics, management and outcome of fractures of the posterior process of the talus. Injury. 2020;51(11):2414-20.

5. Cedell CA. Rupture of the posterior talotibial ligament with the avulsion of a bone fragment from the talus. Acta Orthop Scand. 1974;45(3):454-61.
6. Majeed H, McBride DJ. Talar process fractures: An overview and update of the literature. EFORT Open Rev. 2018;3(3):85-92.

7. Watanabe H, Majima T, Takahashi K, Kawaji H, Takai S. Split Fracture of the Posteromedial Tubercle of the Talus: Case Report and Proposed Classification System. J Foot Ankle Surg. 2017;56(1):187-90.

8. Li CHC, Lui TH. Endoscopically Assisted Reduction and Screw Fixation of Acute Fracture of the Posteromedial Talar Process (Cedell Fracture). Arthrosc Tech. 2020;9(8):e1147-e53.

9. Más Martínez J, Verdú Román C, Martínez Giménez E, Sanz-Reig J, Bustamante Suárez de Puga D, Morales Santías M. Arthroscopic Treatment of a Malunion of a Posteromedial Tubercle Fracture of the Talus. Arthrosc Tech. 2017;6(6):e2107-e10.

10. Ogut T, Seyahi A, Aydingoz O, Bilsel N. A two-portal posterior endoscopic approach in the treatment of a complex talus fracture: a case report. J Am Podiatr Med Assoc. 2009;99(5):443-6. 